\title{
The effects of various lignocelluloses and lignins on physiological responses of a lower termite, Coptotermes formosanus
}

\author{
Didi Tarmadi $^{1,2} \cdot$ Tsuyoshi Yoshimura $^{1} \cdot$ Yuki Tobimatsu $^{3} \cdot$ Masaomi Yamamura $^{3}$. \\ Takuji Miyamoto $^{3}$ - Yasuyuki Miyagawa ${ }^{3}$. Toshiaki Umezawa ${ }^{3}$
}

Received: 8 March 2017/ Accepted: 19 May 2017/Published online: 16 June 2017

(C) The Japan Wood Research Society 2017

\begin{abstract}
We investigated the physiological responses of a lower termite, Coptotermes formosanus, fed on various lignocelluloses and purified lignins (milled-wood lignins, MWLs) from Japanese cedar (softwood), Japanese beech (hardwood), and rice (grass). Termite survival, body mass, and the changes of the symbiotic protists in the hindgut of workers were observed for 4 weeks. The survival, body mass and presence of both Pseudotrichonympha grassii and Holomastigotoides hartmanni in the hindgut of workers fed on rice lignocellulose at the 4 th week of observation were significantly lower than those of the workers fed on Japanese cedar and Japanese beech lignocellulose samples, whereas there was no significant difference in Spirotrichonympha leidyi among all the diets. The three purified MWLs, regardless of their structural differences, did not show any significant differences for the termites' survival or body mass or the survival of all the three protists. The three MWL diets resulted in significantly lower termite survival compared to starvation, although these diets showed no significant effects on body mass or the protist profiles. Overall, lignins are hardly utilized as a nutrient
\end{abstract}

Didi Tarmadi

didi_tarmadi@rish.kyoto-u.ac.jp

1 Laboratory of Innovative Humano-habitability, Research Institute for Sustainable Humanosphere, Kyoto University, Gokasho, Uji, Kyoto 611-0011, Japan

2 Research Center for Biomaterial, Indonesian Institute of Sciences (LIPI), Jl. Raya Bogor KM. 46, Cibinong, Bogor 16911, Indonesia

3 Laboratory of Metabolic Science of Forest Plants and Microorganisms, Research Institute for Sustainable Humanosphere, Kyoto University, Gokasho, Uji, Kyoto 611-0011, Japan source by $C$. formosanus workers and are even rather detrimental to termites when fed on solely.

Keywords Lignocellulose · Lignin · Coptotermes formosanus $\cdot$ Symbiotic protist

\section{Introduction}

Termites represent one of the most abundant and efficient lignocellulose decomposers on earth. Since they are able to degrade lignocellulose, termites have an important place in the carbon cycle in nature [1]. Termites are divided into higher and lower termites based on the presence/absence of symbiotic protists in the termites' hindgut: higher termites lack symbiotic protists in the hindgut, whereas lower termites harbor symbiotic protists in the hindgut $[1,2]$. A lower termite, Coptotermes formosanus is the most important pest of wooden constructions in the world. Three symbiotic protists, Pseudotrichonympha grassii, Holomastigotoides hartmanni, and Spirotrichonympha leidyi were detected in the hindgut of $C$. formosanus; the $P$. grassii used high-molecular weight (MW) cellulose, whereas $H$. hartmanni and S. leidyi used only low-MW cellulose [3, 4]. P. grassii, the largest in size, is spindleshaped, approx. $150-250 \mu \mathrm{m}$ long and $50-100 \mu \mathrm{m}$ wide. $H$. hartmanni, the mid-sized protist, is oval or elliptical shape, approx. $50-150 \mu \mathrm{m}$ long and $30-100 \mu \mathrm{m}$ wide, and $S$. leidyi is the smallest in size, cone-shaped, approx. 20-50 $\mu \mathrm{m}$ long and 10-30 $\mu \mathrm{m}$ wide [3].

Lignocellulose, as the major nutrient source for termites, comprises mainly of cellulose, hemicelluloses and lignin, and it represents the most abundant biomass on earth. Lignin, a heterogeneous aromatic polymer, encrusts cell wall polysaccharides, i.e., cellulose and hemicelluloses, 
and provides them with high resistance to enzymatic degradation [5-7]. Plants have different types of lignins, depending primarily on the plants' taxa. Softwood lignins are composed mainly of guaiacyl (G) units, whereas hardwood lignins consist of $\mathrm{G}$ and syringyl (S) units; both softwood and hardwood lignins also contain a small amount of $p$-hydroxyphenyl $(\mathrm{H})$ units. Like hardwood lignins, lignins from grasses incorporate mostly $\mathrm{G}$ and $\mathrm{S}$ units at comparable levels and often more $\mathrm{H}$ units than softwood and hardwood lignins [5, 8]. In addition, relatively recent studies have revealed that typical grass lignins are highly acylated by $p$-coumarate esters $[9,10]$ and that they also contain a substantial amount of tricin flavonoid units $[11,12]$.

The decomposition of lignocellulose by a termite is a highly coordinated action by the host termite and its gut-resident microbial symbionts [13-15]. To achieve an efficient decomposition of the cell-wall polysaccharides, termites must circumvent the recalcitrant lignin barrier. Studies of lignocellulose decomposition by wood-feeding insects have received significant interest in the last decade [15]. Earlier studies reported that termites are generally unable to decompose lignin polymers during lignocellulose digestion [16], but more recent studies detected structural modifications of lignin polymers in termite digestive systems [17-21].

Although several investigations have described the physiological responses of termites fed on woods [22-24], little information is available regarding the effects of lignins on the physiological activities of wood-feeding insects. Also, there is almost no information regarding the effects of different lignin polymer types from different lignocelluloses on wood-feeding termites. An earlier study by Kyou et al. [25] tested survival of C. formosanus workers fed on an isolated lignin from pine (softwood). In this study, we further closely investigate the influence of three major types of lignin polymers isolated from softwood (Japanese cedar), hardwood (Japanese beech), and grass (rice), on the physiological responses of $C$. formosanus workers as well as their symbiotic protists. The elucidation of physiological responses of wood-feeding insects after exposure to lignins will improve our understanding of the decomposition mechanism of lignocellulose in nature. Such knowledge could also contribute to the development of new technology to convert lignocellulosic biomass to valuable fuels and materials.

\section{Materials and methods}

\section{Lignocellulose components analysis}

Sapwood blocks of Japanese cedar (Cryptomeria japonica) and Japanese beech (Fagus crenata) as well as rice (Oryza sativa) straw were ground into a powder using a ring flaker machine (Wiley mill, Yoshida Seisakusho, Tokyo) and a blender (Vita-Mix Blender, Osaka Chemical, Osaka, Japan), and the particles that passed through a 40-mesh sieve and retained by a 60 -mesh sieve were obtained using a high-speed vibrating sample mill (Iida sieve shaker, Iida Seisakusho, Japan).

The sample meals were soxhlet-extracted with a benzene-ethanol (2:1, v/v) solution for $24 \mathrm{~h} \mathrm{[26].} \mathrm{The} \mathrm{holo-}$ cellulose and lignin contents were measured by using the methods of Wise and a thioglycolic acid lignin assay [27], respectively. The alpha-cellulose was isolated by extracting the holocellulose with a solution of $17.5 \% \mathrm{NaOH}$. The hemicellulose content was then determined by subtracting the cellulose content from the holocellulose content [28]. The chemical component analyses were performed in triplicate.

\section{Preparation of milled-wood lignins (MWLs)}

The isolation of MWLs was conducted as described by Balakshin et al. [29]. Briefly, the extractive-free sample meals were ball-milled using a planetary micro-mill (Pulverisette 7, Fritsch Industrialist, Idar-Oberstein, Germany) with $\mathrm{ZrO}_{2}$ vessels containing $\mathrm{ZrO}_{2}$ ball bearings at $600 \mathrm{rpm}$ for $15 \mathrm{~h}$ (with a $5 \mathrm{~min}$ break for every $10 \mathrm{~min}$ of ball-milling) and then extracted by $96 \%$ dioxin, affording crude MWLs. The crude MWLs were dissolved in $90 \%$ $\mathrm{AcOH}(10 \mathrm{ml} / \mathrm{g}$ sample) and precipitated in water, filtered, washed with water, and then freeze-dried. The resultant powder was again dissolved in a dichloroethane-ethanol $(2: 1, \mathrm{v} / \mathrm{v})$, precipitated in diethyl ether, filtered, washed with diethyl ether, and then dried in vacuo to obtain purified MWLs.

\section{Neutral sugar analysis of MWLs}

Neutral sugar contents in the MWLs were determined as described by Albersheim et al. [30] with a slight modification. Briefly, matrix polysaccharides in the MWLs were decomposed by weak acid hydrolysis with $2 \mathrm{M}$ trifluoroacetic acid $\left(100{ }^{\circ} \mathrm{C}, 5 \mathrm{~h}\right)$. The resulting monomeric sugars were derivatized by the alditol acetate method [31] and quantified by gas chromatography/mass spectrometry (GC-MS; GCMS-QP Plus 2010, Shimadzu, Kyoto, Japan) using myo-inositol as an internal standard. The trifluoroacetic acid-insoluble pellets containing crystalline cellulose were purified by the Updegraff method [32] and further hydrolyzed with sulfuric acid [33]. The glucose concentrations in the obtained solutions were measured using Glucose C-II Test Wako reagent (Wako Pure Chemical, Osaka, Japan) according to manufacturer's instructions. 


\section{Nuclear magnetic resonance (NMR) analysis of MWLs}

The purified MWLs (approx. $15 \mathrm{mg}$ ) were dissolved in $600 \mu 1$ of dimethylsulfoxide (DMSO)- $d_{6}$ and subjected to a NMR analysis on an Avance III 800US system equipped with a cryogenically cooled $5-\mathrm{mm}$ TCI gradient probe (800 MHz, Bruker Biospin, Billerica, MA, USA). The central DMSO solvent peaks $\left(\delta_{\mathrm{C}} / \delta_{\mathrm{H}}: 39.5 / 2.49 \mathrm{ppm}\right)$ were used as an internal reference. Adiabatic heteronuclear single-quantum coherence (HSQC) NMR experiments were carried out using standard implementation ("hsqcgcep.3") with parameters described in the literature [34]. Data processing and analysis used Bruker TopSpin 3.2 software (Bruker Biospin). HSQC plots were obtained with typical matched Gaussian apodization in F2 and squared cosine-bell apodization in F1. For the integration of the lignin aromatic signals (Fig. 1a), $\mathrm{C}_{2}-\mathrm{H}_{2}$ correlations from $\mathrm{G}$ units $(\mathrm{G})$ and $\mathrm{C}_{2}-\mathrm{H}_{2} / \mathrm{C}_{6}-\mathrm{H}_{6}$ correlations from $\mathrm{S}$ units (S), $\mathrm{H}$ units $(\mathrm{H})$, and $p$-coumarate units $(p \mathrm{CA})$, and $\mathrm{C}_{2^{\prime}}-\mathrm{H}_{2^{\prime}}$ l $\mathrm{C}_{6^{\prime}}-\mathrm{H}_{6^{\prime}}$ correlations from tricin units (T), were used, and the $\mathrm{S}, \mathrm{H}, p \mathrm{CA}$, and $\mathrm{T}$ integrals were logically halved [35]. For the integrations of the lignin inter-monomeric linkages (Fig. 1b), well-resolved $\mathrm{C}_{\alpha}-\mathrm{H}_{\alpha}$ contours from I, I', II, II', III, III', IV and V units were integrated and the III and III' integrals were logically halved [34, 35].

\section{Termite survival and body mass}

Sapwood blocks of Japanese cedar and Japanese beech [10 $(\mathrm{R}) \times 10(\mathrm{~T}) \times 10(\mathrm{~L}) \mathrm{mm}]$ or 25 -mm-long rice straws were put into an acrylic cylinder with a plaster bottom. Approximately $100 \mathrm{mg}$ of purified MWL was placed in a plastic cup (20 $\mathrm{mm}$ dia., $10 \mathrm{~mm}$ deep), and this container was set on the center of an acrylic cylinder with a plaster bottom ( $65 \mathrm{~mm}$ dia., $60 \mathrm{~mm}$ high). Fifty workers and five soldiers of $C$. formosanus were obtained from a laboratory colony at the Deterioration Organisms Laboratory (DOL), Research Institute for Sustainable Humanosphere (RISH), Kyoto University, were fed on the various diets. The number of live workers was recorded and balanced weekly for 4 weeks. The termite survival and body mass observations were performed in triplicate.

\section{Changes of protist fauna in the hindguts of the $C$.formosanus workers}

In this manner, 130 workers and 13 soldiers of $C$. formosanus were fed on the lignocellulose and MWL diets as described above. Every week, 10 workers were randomly selected from each container, and their hindguts were pulled out from the posterior ends and disintegrated into pieces by using fine forceps. The contents were then suspended in $0.4 \% \mathrm{NaCl}$. The hindgut pieces were macerated gently in the solution to facilitate the release of protozoa. The species of protozoa were identified using a digital microscope (VHX-5000, Keyence, Osaka, Japan) [4]. These observations were performed in triplicate.

\section{Statistical analysis}

Differences in the termites' survival and body mass and the presence of protists in the hindgut of the workers fed on various lignocelluloses and MWLs were analyzed using SPSS ver. 23 software (IBM, Armonk, NY). Significant differences between means were identified by Tukey's post hoc test. Significance levels were set at $P<0.05$.

\section{Results and discussion}

\section{Characterization of lignocellulose and MWL dietary samples}

At the onset of this study, we first characterized the lignocellulose and lignin samples used as diets for termites. The composition of the three major lignocellulose components, i.e., cellulose, hemicelluloses, and lignin in Japanese cedar (softwood), Japanese beech (hardwood), and rice (grass) samples is summarized in Table 1. Polysaccharides, i.e., cellulose and hemicelluloses, accounted for $67-77 \%$ of the three lignocellulose samples. The lignin content in the rice (approx. 15\%) was somewhat lower than those in the two wood samples (20-24\%), which is in line with previous reports [36, 37]. It is conceivable that our rice lignocellulose sample also contained amounts of minerals such as silica [38, 39]. Purified lignin samples were isolated as MWLs by typical procedures [29], and their purity and structure were checked by sugar analysis and 2D-NMR. The sugar analysis data indicated that the purities of all three MWLs were reasonably high (>85\%) (Table 2). All of the MWLs contained approx. $10 \%$ residual cellulosic glucans. The rice MWL additionally contained approx. 5\% hemicellulosic sugars, mainly composed of xylose, whereas the MWLs from Japanese cedar and Japanese beech had almost negligible amounts of hemicellulosic sugars; the difference might be due to that lignin and hemicelluloses are cross-linked more highly in grass lignocellulose than in softwood and hardwood lignocelluloses [9].

Our HSQC NMR data firmly established that the three MWLs, respectively display the characteristics of the three major lignin types in nature. As revealed by the aromatic signals in the HSQC spectra (Fig. 1a), the MWL from Japanese cedar is a typical softwood lignin composed of only $G$ units, whereas the MWLs from the hardwood 
(a) Aromatic regions

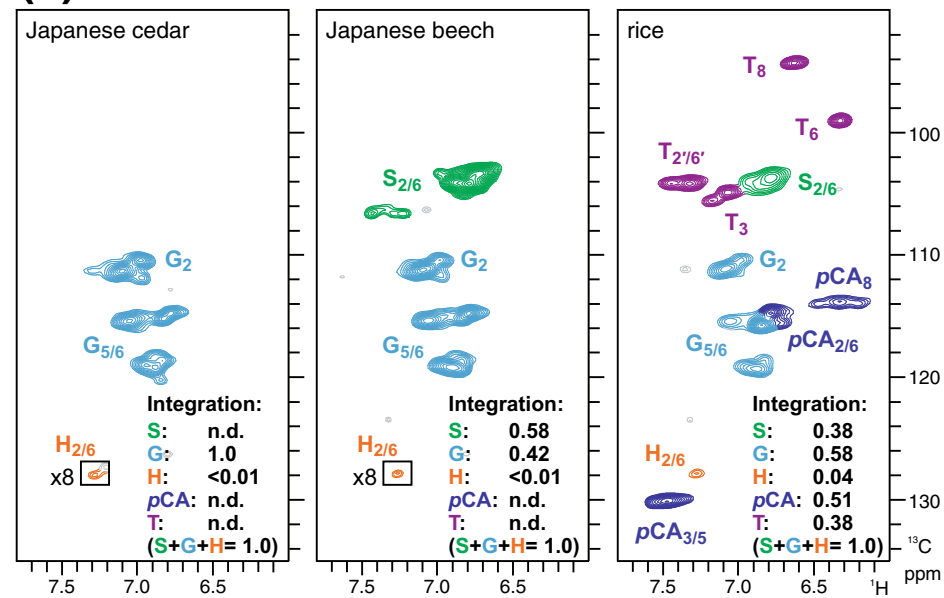

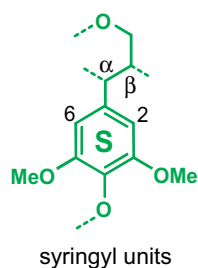
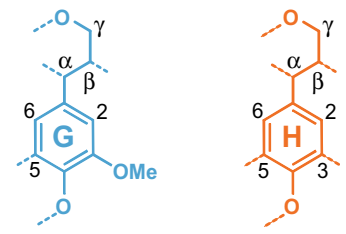

syringyl units

guaiacyl units

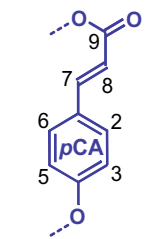

p-coumarate units

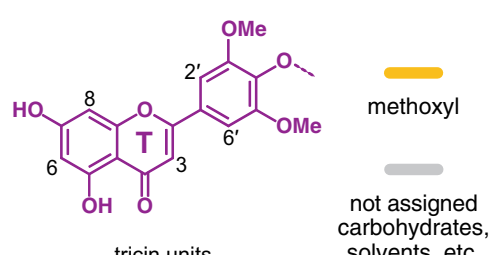

tricin units

(b) Aliphatic regions
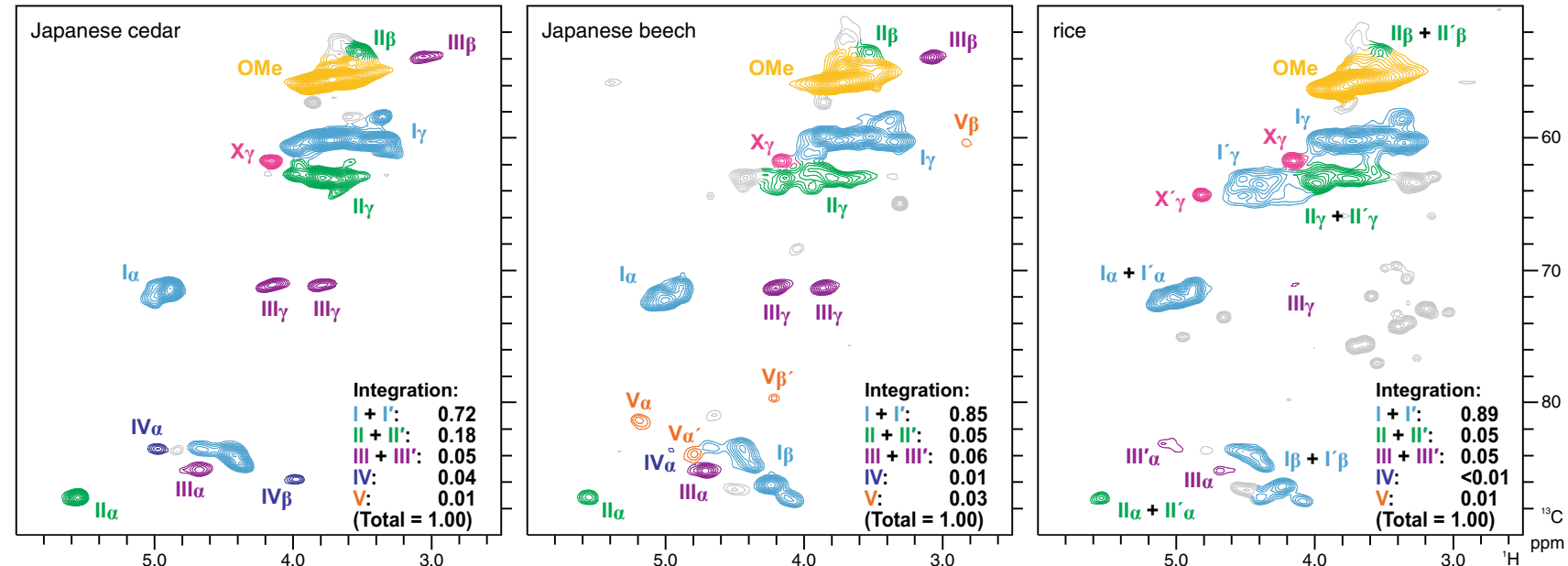

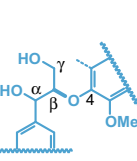

I

$(\mathrm{\gamma}-\mathrm{OH})$

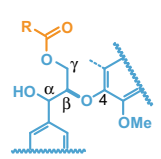

I'

$\beta-\mathrm{O}-4$

( $\gamma$-acylated $)$
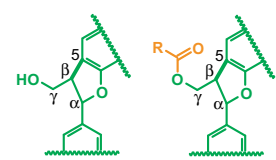

II

$(\gamma-\mathrm{OH})$

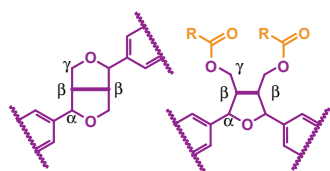

II'

$\beta-5$

$(\gamma$-acylated $)$

III

$\beta-\beta$
$(\gamma-O-\alpha)$
III'

$(\gamma$-acylated $)$

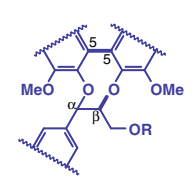

IV dibenzodioxocin $5-5 / \beta-0-4$

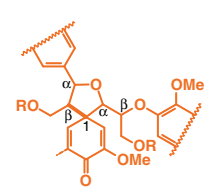

V cinnamyl alcoho end-units
( $\mathrm{y}$-acylated $)$
Fig. 1 Aromatic (a) and aliphatic (b) sub-regions of short-range ${ }^{1} \mathrm{H}-{ }^{13} \mathrm{C}$ correlation (HSQC) NMR spectra of milled-wood lignins isolated from Japanese cedar, Japanese beech, and rice lignocellulose samples. Volume integrals are given for the major lignin aromatic and side-chain structures that are color-coded to match their assignments in the spectrum. Boxes labeled $\times 8$ indicate regions that are vertically scaled eightfold
Table 1 Cellulose,

hemicellulose and lignin composition in Japanese cedar, Japanese beech, and rice lignocellulose diet samples

\begin{tabular}{llll}
\hline Samples & \multicolumn{2}{l}{ Lignocellulose composition $(\%)^{\mathrm{a}}$} \\
\cline { 2 - 4 } & Cellulose & Hemicelluloses & Lignin \\
\hline Japanese cedar sapwood & $44.14 \pm 0.46$ & $30.16 \pm 0.62$ & $23.92 \pm 2.00$ \\
Japanese beech sapwood & $41.48 \pm 0.72$ & $35.77 \pm 0.67$ & $19.60 \pm 0.37$ \\
Rice straw & $39.52 \pm 0.97$ & $26.94 \pm 2.27$ & $15.02 \pm 0.47$ \\
\hline
\end{tabular}

$S D$ standard deviation

${ }^{\text {a }}$ Values are mean $\pm \mathrm{SD}(n=3)$ 
Table 2 Carbohydrate contents and composition in MWL diet samples prepared from Japanese cedar, Japanese beech, and rice

\begin{tabular}{llll}
\hline Carbohydrates & \multicolumn{3}{l}{ Content $(\%)^{\mathrm{a}}$} \\
\cline { 2 - 4 } & Japanese cedar & Japanese beech & Rice \\
\hline Hemicellulosic sugars & & & \\
Arabinan & $0.03 \pm 0.00$ & $0.05 \pm 0.00$ & $0.75 \pm 0.07$ \\
Xylan & $0.04 \pm 0.00$ & $0.95 \pm 0.03$ & $4.39 \pm 0.50$ \\
Mannan & $0.16 \pm 0.01$ & $0.16 \pm 0.01$ & $0.21 \pm 0.01$ \\
Galactan & $0.11 \pm 0.00$ & $0.11 \pm 0.01$ & $0.18 \pm 0.01$ \\
Glucan & $0.07 \pm 0.01$ & $0.31 \pm 0.01$ & $1.31 \pm 0.13$ \\
Cellulosic glucan & $7.99 \pm 1.47$ & $9.50 \pm 2.99$ & $9.14 \pm 1.49$
\end{tabular}

$S D$ standard deviation

a Values are mean $\pm \mathrm{SD}(n=3)$

b Determined as trifluoroacetic acid-hydrolyzable sugars

Japanese beech and grass rice are mixtures of $\mathrm{G}$ and $\mathrm{S}$ units. The volume integration of the contour signals allowed reasonable quantifications of the $S / G$ unit ratios 1.38 and 0.66 in the Japanese beech and rice MWLs, respectively. As a typical lignin in grasses, the spectrum of rice MWL displayed more intense signals from $\mathrm{H}$ units $(\mathrm{H})$ than those in the spectra of the Japanese cedar and Japanese beech MWLs, and the rice MWL also displayed clear signals from $p$-coumarate $(p \mathrm{CA})$ and tricin $(\mathrm{T})$ units [35], which were totally absent in the Japanese cedar and Japanese beech MWL spectra. The aliphatic regions of the HSQC spectra (Fig. 1b) resolve most of the correlations for the various lignin interunit linkage types. In all of the MWL spectra, the most predominant lignin linkage type was $\beta-O-4$ units (I and $\mathrm{I}^{\prime}$ ) with lesser amounts of $\beta-5$ (II and $\mathrm{II}^{\prime}$ ), $\beta-\beta$ (III and $\mathrm{III}^{\prime}$ ), 5-5/ $\beta-O-4$ (IV), and $\beta-1$ (V) units. Compared to the Japanese cedar MWL, the proportions of $\beta-O-4$ units were apparently higher and those of $\beta-5$ and 5-5/ $\beta-O-4$ units were conversely lower in the Japanese beech and rice MWLs. Such shifts of lignin linkage distributions are typical when lignin incorporates more $\mathrm{S}$ units than $\mathrm{G}$ units [40, 41]. The appearance of characteristic signals from acylated lignin units $\left(\mathrm{I}^{\prime}, \mathrm{II}^{\prime}\right.$, and $\mathrm{III}^{\prime}$ ) in the rice MWL spectrum confirmed that rice MWL, as a typical grass lignin, is highly acylated, presumably by $p$-coumarates $[9,10,35]$.

\section{Termite survival and body mass}

The periodic changes in termite survival are summarized in Fig. 2. The survival of termites fed on lignocelluloses from Japanese cedar, Japanese beech, and rice showed no significant differences at the 1 st-week observation $(F=7$, $P=0.27)$ at 100,99 , and $98 \%$, respectively, or at 2 weeks $(F=3, P=0.131)$ at 99,95 , and $91 \%$, respectively. Interestingly, at 3 weeks, the survival of the termites fed on rice was significantly lower than that of the termites fed on Japanese cedar and Japanese beech $(F=76, P<0.05)$, and there was no significant difference in survival between the Japanese cedar and Japanese beech groups $(F=1$, $P=0.374$ ) at 92,91 , and $76 \%$, respectively. The results at the end of the experiment (4 weeks) were similar to those at 3 weeks, in that the survival rate of the termites fed on rice was significantly lower than those of the termites fed on Japanese cedar and Japanese beech $(F=116$, $P<0.05$ ), with no significant difference between the Japanese cedar and Japanese beech groups $(F=2$, $P=0.230$ ) at 87,85 , and $70 \%$, for Japanese cedar, Japanese beech, and rice, respectively. These results clearly suggest that wood was more nutritious than rice.

Termites use the carbon source from woods as a nutrient to maintain their survival [4, 42, 43]. Termite workers break down wood with their mandibles and foregut gizzard. Thus, endogenous cellulases and a variety of cellulolytic enzymes secreted by the symbiotic microorganisms community in the gut coordinately digest carbohydrates and efficiently convert them into acetates and other nutrients as the main energy and carbon source for the host [13, 15]. As mentioned above, termites are divided into higher and lower termites based on symbiotic protists in the hindgut. The lower termites possess symbiotic protists; higher termites lack symbiotic protists $[2,3]$. The lower termites are well known as wood-feeding insects [43, 44]. In nature, grasses can also be decomposed by the higher termites (grass-feeding termites) [45, 46].

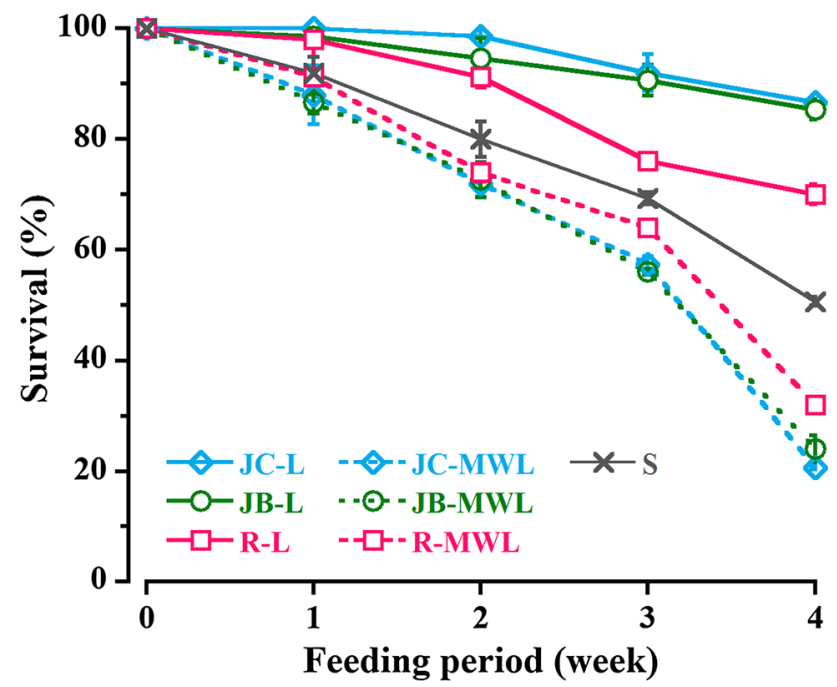

Fig. 2 Changes in the survival rate of $C$. formosanus workers fed on various lignocelluloses and isolated lignins (MWLs). Error bars represent standard deviation $(n=3)$. $J C$ - $L$ Japanese cedar lignocellulose, $J B-L$ Japanese beech lignocellulose, $R-L$ rice lignocellulose, $J C-M W L$ Japanese cedar MWL, $J B-M W L$ Japanese beech MWL, $R$ $M W L$ rice MWL, $S$ starvation control group 
As shown in Fig. 2, the average of the workers' survival rate in the three lignocellulose groups (Japanese cedar, Japanese beech, and rice) compared to starvation were significantly different at the 1st-week $(F=28.3, P<0.05)$ and 2nd-week ( $F=17.5, P=0.001)$ observations at $92 \%$ for the lignocellulose groups and $80 \%$ for starvation. Surprisingly, at the 3rd-week, the survival rate was not significantly different between rice and starvation, whereas the survival of the starvation group was significantly lower than those of the termites fed on Japanese cedar and Japanese beech $(F=29, P<0.05)$ at $69 \%$ for starvation. Finally, at the 4th-week, we observed that the survival rate of the starvation group was significantly lower than those of the groups fed on Japanese cedar, Japanese beech, and rice $(F=47, P<0.05)$ at $51 \%$ for starvation. These results suggest that the lower termite $C$. formosanus can still utilize some of carbohydrate components in rice.

Figure 2 also shows the survival rates of workers fed on the three MWLs from Japanese cedar, Japanese beech, and rice. Our statistical analysis revealed that at 1,2,3, and 4 weeks, there were no significant differences in survival among the three MWLs from Japanese cedar, Japanese beech, and rice. Subsequently, compared to starvation, the survival rates for the three MWL groups were also not significantly different at 1 week $(F=1.6, P=0.271)$, 2 weeks $(F=1.6, P=0.269)$, and 3 weeks $(F=3.3$, $P=0.078)$. However, at the end of the observation at 4 weeks, the statistical analysis showed that the survival rates of the three MWL groups were significantly lower than that of the starvation group $(F=14, P=0.002)$. These results revealed that the three MWLs from Japanese cedar, Japanese beech, and rice were not nutritious for $C$. formosanus, and rather were somewhat detrimental when the termites were fed on each MWL solely. It has been recognized that the lower termites digest carbohydrates as the main nutrient source with the aid of a symbiotic protistan community in their gut [13, 14]. Based on the metatranscriptomes of the symbiotic protistan community in the hindgut of $C$. formosanus, no potential lignin degradation enzymes have yet been found [47]. However, the workers of $C$. formosanus often digest nest materialsespecially in the winter season, which provides digestive residues (rich in lignin) and soil [3].

Figure 3 shows the periodic changes in the body mass of the workers fed on the lignocelluloses and MWLs. There was no significant difference in the body mass of the workers among the three lignocellulose groups at 1 week $(F=0.8, P=0.500), 2$ weeks $(F=3.5, P=0.100)$, and 3 weeks $(F=2.4, P=0.169)$. Interestingly, the components in rice apparently affect the body mass of termites, as at 4 weeks the body mass values of the termites were significantly lower than those of the termites fed on Japanese cedar and Japanese beech sapwoods $(F=46.3$,

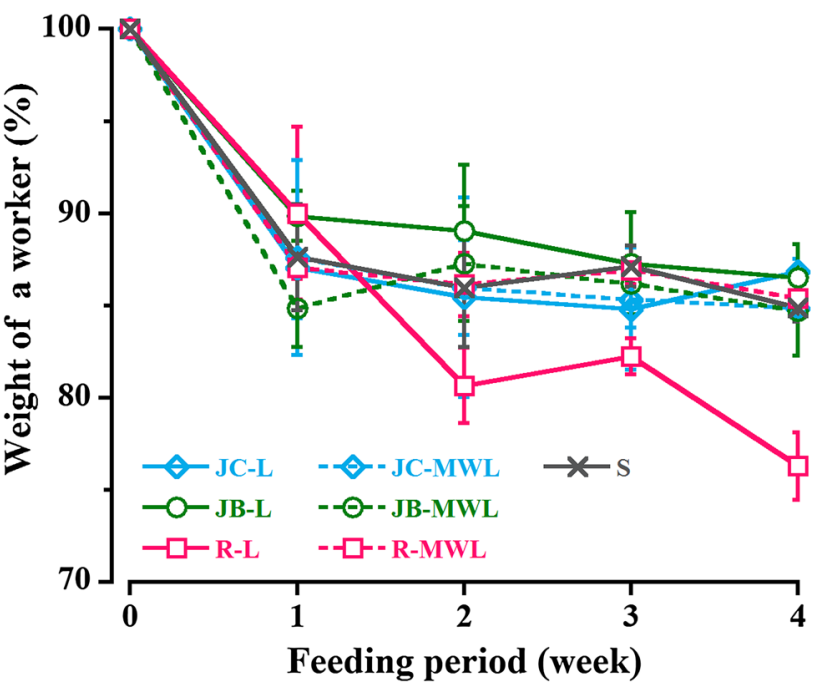

Fig. 3 Changes in the body mass of $C$. formosanus workers fed on various lignocelluloses and isolated lignins (MWLs). Error bars represent standard deviation $(n=3)$. $J C$ - $L$ Japanese cedar lignocellulose, $J B-L$ Japanese beech lignocellulose, $R-L$ rice lignocellulose, $J C-M W L$ Japanese cedar MWL, $J B-M W L$ Japanese beech MWL, $R$ $M W L$ rice MWL, $S$ starvation control group

$P<0.05)$, while no significant difference was observed between the Japanese cedar and Japanese beech groups $(F=0.1, P=0.831)$, at $86.9,86.5$, and $76.3 \%$ of the relative body mass for Japanese cedar, Japanese beech, and rice, respectively. Compared to the starvation group, the body mass values of the termites in the three lignocellulose groups were not significantly different at 1,2, and 3 weeks. At 4 weeks, the body mass values of the termites fed on rice were significantly lower than those of the starvationgroup termites $(F=214, P<0.05$ ), whereas no significant difference was observed between both sapwood groups (Japanese cedar and Japanese beech) and the starvation group $(F=1.4, P=0.321)$. These results suggest that $C$. formosanus use part of the carbohydrates in rice as nutrients as discussed above, but this nutrient supply was insufficient for termites, resulting in the decreased body mass.

The body mass values of the termites fed on the three MWLs from Japanese cedar, Japanese beech, and rice (Fig. 3) were not significantly different at 1, 2, 3 or 4 weeks. In addition, all three MWL groups and the starvation group did not show any significant differences at 1 , 2,3 or 4 weeks. These results further support the possibility that termites hardly use lignins as a nutrient source. Given that the termite survival rates were significantly lower in the MWL groups compared to starvation (Fig. 2), cannibalism [48] may help termites maintain their body mass when being fed on non-nutritious food and even possibly toxic lignin polymers. As described above, even with no significant differences between the workers tested 
with the three different MWL diets, both the survival rate and body mass of the workers fed with rice grass lignocellulose were significantly lower than those with Japanese cedar and Japanese beech wood lignocelluloses. This suggests that some components other than lignin in grass lignocellulose are negatively affecting the physiology of $C$. formosanus workers. Although further investigations are needed, we suspect that minerals such as silica relatively rich in grass lignocellulose [38, 39] could be responsible for this phenomenon.

\section{Intestinal protist profiles}

Lastly, we investigated the changes in the protist profiles in the hindguts of the worker termites fed on the lignocelluloses and MWLs (Table 3). We found that all the three protists (i.e., P. grassii, H. hartmanni, and S. leidyi) [4] remained in the hindguts of all of the workers fed on the three lignocelluloses at the 1st-week observation. However, feeding on rice resulted in a loss of $P$. grassii in $17 \%$ of the workers and $47 \%$ of the workers at the 2 nd- and 3rdweek observations, respectively, whereas $H$. hartmanni and $S$. leidyi remained in all of the workers at these time points.

At the end of the observation (4 weeks), the rice feeding had resulted in a loss of $P$. grassii in $73 \%$ of the workers, $H$. hartmanni in $17 \%$ of the workers, and S. leidyi in $7 \%$ of the workers (Table 3). In contrast, all three protists remained in all of the workers fed on Japanese cedar and Japanese beech even at 4 weeks. There were significant differences between both sapwoods and rice for $P$. grassii $(F=484, \quad P<0.05), \quad$ and $H$. hartmanni $(F=25$, $P=0.001)$, but not for $S$. leidyi $(F=1, P=0.422)$. These results are in line with our previous observation of termite survival (Fig. 2) and body mass (Fig. 3), and with the fact that lower termites including $C$. formosanus prefer woods compared to grasses as diets; as discussed above, it is plausible that minerals rich in grass lignocellulose could be negatively affecting the survival of the two major protists. Nevertheless, our results demonstrated that the presence of all three of these protists in workers fed on rice was significantly higher than those in the starved workers (Table 3), suggesting that the protists still can utilize carbohydrates in rice as a nutrient source.

On the other hand, after termites were fed on the three MWLs from Japanese cedar, Japanese beech, and rice for 1 week, $P$. grassii disappeared from all of the workers, whereas $H$. hartmanni and $S$. leidyi remained in all workers. There was no significant difference among the three MWLs for all protists at 2 and 3 weeks. At the end of the 4th-week observation, $67-77 \%$ of the workers had lost H. hartmanni and $40-50 \%$ of the workers had lost S. leidyi (Table 3). No significant difference regarding the survival

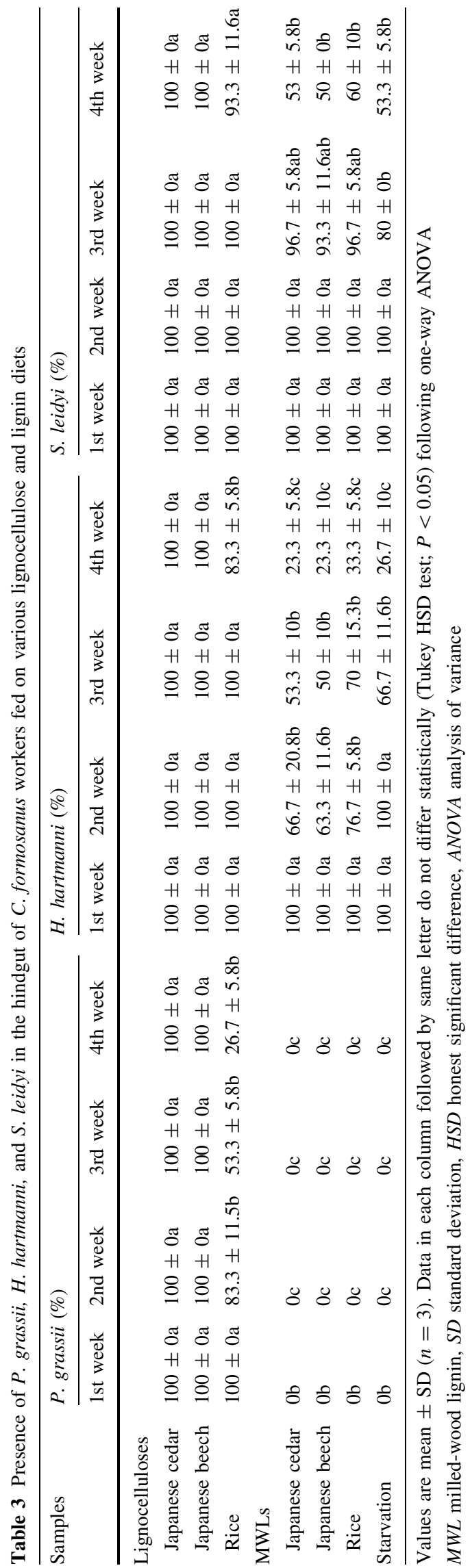


of protists fed on the three MWLs was observed at the end of the observation for $P$. grassii (lost in all workers), $H$. hartmanni $(F=3, P=0.125)$, and $S$. leidyi $(F=1.8$, $P=0.252$ ). In addition, there was no significant difference between the three MWLs and starvation for all of the protists at the end of the observation: $P$. grassii (lost in all workers), $H$. hartmanni $(F=2, P=0.193)$, and $S$. leidyi ( $F=1.3, P=0.349)$. Collectively, our data suggest that all the three types of lignin polymers, regardless of their structural differences, had no effect on the survival of protists in the hindgut of workers of C. formosanus.

\section{Conclusions}

Overall, our data suggest that different types of lignins from softwood, hardwood, and grass did not significantly affect the physiological responses of $C$. formosanus and its symbiotic protists. The results of this study established that lignins are hardly utilized as a nutrient source by $C$. formosanus workers and even are rather somewhat detrimental to termites when fed on solely. We also observed that some components other than lignins (possibly minerals in grass lignocellulose) negatively affect the physiological responses of $C$. formosanus and its major symbiotic protists. These new findings contribute to the basic knowledge on the mechanism of lignocellulose decomposition by wood-feeding insects. We plan to investigate the nutritional effects of combinations of carbohydrates and lignins on the physiological activities of $C$. formosanus and its protists to identify the exact role of lignin in termites.

Acknowledgements We thank Mr. Akio Adachi and Dr. Izumi Fujimoto (RISH, Kyoto University) for preparing the wood samples and termites, the Shiga Prefecture Agricultural Technology Promotion Center for providing rice straw samples, Prof. Takashi Watanabe and Dr. Hiroshi Nishimura (RISH, Kyoto University) for their support in the preparation of the MWL samples, and Prof. Hironori Kaji and Ms. Ayaka Maeno [Institute for Chemical Research (ICR) of Kyoto University] for their support in the NMR experiments. A part of this study was conducted using the facilities in the DASH/FBAS at RISH, Kyoto University, and the NMR spectrometer in the JURC at ICR, Kyoto University.

\section{References}

1. Brune A (2014) Symbiotic digestion of lignocellulose in termite guts. Nat Rev Microbiol 12:168-180

2. Lo N, Eggleton P (2011) Termite phylogenetics and co-cladogenesis with symbionts. In: Bignell DE, Roisin Y, Lo N (eds) Biology of termites: a modern synthesis. Springer, Dordrecht, pp 27-50

3. Yoshimura $\mathrm{T}$ (1995) Contribution of the protozoan fauna to nutritional physiology of the lower termite, Coptotermes formosanus Shiraki (Isoptera: Rhinotermitidae). Wood Res 82:68-129
4. Tanaka H, Aoyagi H, Shina S, Dodo Y, Yoshimura T, Nakamura $\mathrm{R}$, Uchiyama H (2006) Influence of the diet components on the symbiotic microorganisms community in hindgut of Coptotermes formosanus Shiraki. Appl Microbiol Biotechnol 71:907-917

5. Boerjan W, Ralph J, Baucher M (2003) Lignin biosynthesis. Ann Rev. Plant Biol 54:519-546

6. Jackson LA, Shadle GL, Zhou R, Nakashima J, Chen F, Dixon RA (2008) Improving saccharification efficiency of alfalfa stems through modification of the terminal stages of monolignol biosynthesis. Bioenerg Res 1:180-192

7. Tobimatsu Y, Elumalai S, Grabber JH, Davidson CL, Pan X, Ralph J (2012) Hydroxycinnamate conjugates as potential monolignol replacements: in vitro lignification and cell wall studies with rosmarinic acid. ChemSusChem 5:676-686

8. Bonawitz ND, Chapple C (2010) The genetics of lignin biosynthesis: connecting genotype to phenotype. Annu Rev Genet 44:337-363

9. Ralph J (2010) Hydroxycinnamates in lignification. Phytochem Rev 9:65-83

10. Petrik DL, Karlen SD, Cass CL, Padmakshan D, Lu F, Liu S, Le Bris P, Antelme S, Santoro N, Wilkerson CG, Sibout R, Lapierre C, Ralph J, Sedbrook JC (2014) p-Coumaroyl-CoA: monolignol transferase (PMT) acts specifically in the lignin biosynthetic pathway in Brachypodium distachyon. Plant J 77:713-726

11. Lan W, Lu F, Regner M, Zhu Y, Rencoret J, Ralph SA, Zakai UI, Morreel K, Boerjan W, Ralph J (2015) Tricin, a flavonoid monomer in monocot lignification. Plant Physiol 167:1284-1295

12. Lan W, Rencoret J, Lu F, Karlen SD, Smith BG, Harris PJ, del Río JC, Ralph J (2016) Tricin-lignins: occurrence and quantitation of tricin in relation to phylogeny. Plant J 88:1046-1057

13. Hongoh Y (2011) Toward the functional analysis of uncultivable, symbiotic microorganisms in the termite gut. Cell Mol Life Sci 68:1311-1325

14. Ohkuma M (2003) Termite symbiotic systems: efficient bio-recycling of lignocellulose. App Microbiol Biotechnol 61:1-9

15. Ni J, Tokuda G (2013) Lignocellulose-degrading enzymes from termites and their symbiotic microbiota. Biotechnol Adv 31:838-850

16. Breznak JA, Brune A (1994) Role of microorganisms in the digestion of lignocellulose by termites. Annu Rev Entomol 39:453-487

17. Geib SM, Filley TR, Hatcher PG, Hoover K, Carlson JE, del Mar Jimenez-Gasco M, Nakagawa-Izumi A, Sleighter RL, Tien M (2008) Lignin degradation in wood-feeding insects. Proc Natl Acad Sci USA 105:12932-12937

18. Hyodo F, Azuma JI, Abe T (1999) Estimation of effect of passage through the gut of a lower termite, Coptotermes formosanus Shiraki, on lignin by solid-state CP/MAS ${ }^{13} \mathrm{C}$ NMR. Holzforschung 53:244-246

19. Katsumata KS, Jin Z, Hori K, Iiyama K (2007) Structural changes in lignin of tropical woods during digestion by termite, Cryptotermes brevis. J Wood Sci 53:419-426

20. Ke J, Singh D, Chen S (2011) Aromatic compound degradation by the wood-feeding termite Coptotermes formosanus (Shiraki). Int Biodeterior Biodegrad 65:744-756

21. Ke J, Laskar DD, Chen S (2013) Varied lignin disruption mechanisms for different biomass substrates in lower termite. Renew Energ 50:1060-1064

22. Fei H, Henderson G (2002) Formosan subterranean termite (Isoptera: Rhinotermitidae) wood consumption and worker survival as affected by temperature and soldier proportion. Environ Entomol 31:509-514

23. Hapukotuwa NK, Grace JK (2011) Preferences of Coptotermes formosanus Shiraki and Coptotermes gestroi (Wasmann) (Blattodea: Rhinotermitidae) among three commercial wood species. Insects 2:499-508 
24. Nakayama T, Yoshimura T, Imamura Y (2005) Feeding activities of Coptotermes formosanus Shiraki and Reticulitermes speratus (Kolbe) as affected by moisture content of wood. J Wood Sci 51:60-65

25. Kyou K, Watanabe T, Yoshimura T, Takahashi M (1996) Lignin modification by termite and its symbiotic protozoa. Wood Res 83:50-54

26. Waite R, Gorrod ARN (1959) The structural carbohydrates of grasses. J Sci Food Agric 10:308-317

27. Suzuki S, Suzuki Y, Yamamoto N, Hattori T, Sakamoto M, Umezawa T (2009) High-throughput determination of thioglycolic acid lignin from rice. Plant Biotechnol 26:337-340

28. Kusumah SS, Umemura K, Yoshioka K, Miyafuji H, Kanayama K (2016) Utilization of sweet sorghum bagasse and citric acid for manufacturing of particleboard I: effects of pre-drying treatment and citric acid content on the board properties. Ind Crop Prod 84:34-42

29. Balakshin M, Capanema E, Gracz H, Chang HM, Jameel H (2011) Quantification of lignin-carbohydrate linkages with highresolution NMR spectroscopy. Planta 233:1097-1110

30. Albersheim P, Nevins DJ, English PD, Karr A (1967) A method for the analysis of sugars in plant cell-wall polysaccharides by gas-liquid chromatography. Carbohydr Res 5:340-345

31. Hayashi T (1989) Measuring $\beta$-glucan deposition in plant cell walls. In: Linskens HF, Jackson JF (eds) Modern methods of plant analysis: plant fibers, 10. Springer, Berlin, pp 138-160

32. Updegraff DM (1969) Semimicro determination of cellulose in biological materials. Anal Biochem 320:420-424

33. Yamamura M, Noda S, Hattori T, Shino A, Kikuchi J, Takabe K, Tagane S, Gau M, Uwatoko N, Mii M, Suzuki S, Shibata D, Umezawa T (2013) Characterization of lignocellulose of Erianthus arundinaceus in relation to enzymatic saccharification efficiency. Plant Biotechnol 30:25-35

34. Mansfield SD, Kim H, Lu F, Ralph J (2012) Whole plant cell wall characterization using solution-state 2D NMR. Nat Protoc 7:1579-1589

35. Lam PY, Tobimatsu Y, Takeda Y, Suzuki S, Yamamura M, Umezawa T, Lo C (2017) Disrupting flavone synthase II alters lignin and improves biomass digestibility. Plant Physiol. doi:10. 1104/pp. 16.01973

36. Ehara K, Takada D, Saka S (2005) GC-MS and IR spectroscopic analyses of the lignin-derived products from softwood and hardwood treated in supercritical water. J Wood Sci 51:256-261
37. Zhu S, Wu Y, Yu Z, Wang C, Yu F, Jin S, Ding Y, Chi R, Liao J, Zhang Y (2006) Comparison of three microwave/chemical pretreatment processes for enzymatic hydrolysis of rice straw. Biosyst Eng 93:279-283

38. Jenkins BM, Baxter LL, Miles TR Jr, Miles TR (1998) Combustion properties of biomass. Fuel Process Technol 54:17-46

39. Binod P, Sindhu R, Singhania RR, Vikram S, Devi L, Nagalakshmi S, Kurien N, Sukumaran RK, Pandey A (2010) Bioethanol production from rice straw: an overview. Bioresour Technol 101:4767-4774

40. Stewart JJ, Akiyama T, Chapple C, Ralph J, Mansfield SD (2009) The effects on lignin structure of overexpression of ferulate 5-hydroxylase in hybrid poplar. Plant Physiol 150:621-635

41. Wagner A, Tobimatsu Y, Phillips L, Flint H, Geddes B, Lu F, Ralph J (2015) Syringyl lignin production in conifers: Proof of concept in a pine tracheary element system. Proc Natl Acad Sci USA 112:6218-6223

42. Katsumata N, Yoshimura T, Tsunoda K, Imamura Y (2007) Termite feeding preference to four wood species after gamma irradiation. J Wood Sci 53:361-364

43. Morales-Ramos JA, Rojas MG (2001) Nutritional ecology of the formosan subterranean termite (Isoptera: Rhinotermitidae): feeding response to commercial wood species. J Econ Entomol 94:516-523

44. Su NY, Tamashiro M (1986) Wood-consumption rate and survival of the Formosan subterranean termite (Isoptera: Rhinotermitidae) when fed one of six woods used commercially in Hawaii. Proc Hawaii Entomol Soc 26:109-113

45. Ohiagu C (1979) A quantitative study of seasonal foraging by the grass harvesting termite, Trinervitermes geminatus (Wasmann) (Isoptera, Nasutitermitinae) in Southern Guinea Savanna, Mokwa, Nigeria. Oecologia 40:179-188

46. Jouquet P, Traoré S, Choosai C, Hartmann C, Bignell D (2011) Influence of termites on ecosystem functioning. Ecosystem services provided by termites. Eur J Soil Biol 47:215-222

47. Xie L, Zhang L, Zhong Y, Liu N, Long Y, Wang S, Zhou X, Zhou Z, Huang Y, Wang Q (2012) Profiling the metatranscriptome of the protistan community in Coptotermes formosanus with emphasis on the lignocellulolytic system. Genomics 99:246-255

48. Cook SF, Scott KG (1933) The nutritional requirements of Zootermopsis (Termopsis) angusticollis. J Cell Comp Physiol 4:95-110 